\title{
Évaluation préliminaire d'un stage d'externat expérimental en médecine générale à la faculté de médecine de Tours
}

\author{
Preliminary evaluation of an experimental clerkship in General Practice \\ at the Faculty of medicine of Tours
}

\section{Cécile Renoux, Anne Marie Lehr-Drylewicz, Dominique Huas et Jean Pierre Lebeau}

Département universitaire de médecine générale, Faculté de médecine, Université François Rabelais, 10 boulevard Tonnellé - BP 3223 - 37032 Tours Cedex 1, France

Manuscrit reçu le 7 décembre 2008; commentaires éditoriaux formulés aux auteurs le 17 juin 2009; accepté pour publication le $1^{\text {er }}$ octobre 2009

\section{Mots clés :}

Médecine générale ; stage ;

externes ;

vécu

Keywords:

General practice;

training;

clerkship students;

experience
Résumé - Contexte : En 2007, le département de médecine générale de Tours a expérimenté un stage en médecine générale de 2 semaines, à temps plein, pour tous les étudiants en $3^{\mathrm{e}}$ année du deuxième cycle (DCEM3). Dans le cadre de son évaluation, cette étude avait pour objectif d'explorer le vécu des externes de leur stage. Méthodes : Étude qualitative explorant le vécu des externes par quatre focus group auprès de 29 des 104 externes ayant effectué un stage entre juillet et septembre 2007. Une retranscription écrite intégrale et une analyse interprétative des verbatim ont été effectuées. Résultats : Le stage a été apprécié dans sa forme et sa durée. Les externes ont découvert la spécialité dans sa diversité et sa pratique et l'exercice ambulatoire. Ils ont appréhendé avec plaisir de nombreuses spécificités de la médecine générale : la globalité de la prise en charge, la relation médecin-patient, la démarche diagnostique en ambulatoire, les visites à domicile... Ce stage a été jugé utile dans l'orientation professionnelle des étudiants, leur permettant un choix en connaissance de cause. Conclusion : Le stage a été bien vécu par les externes. Cette étude a mis en évidence leur méconnaissance de la médecine générale et de l'univers ambulatoire. Cette première expérience, si les conditions humaines et matérielles sont réunies, sera renouvelée du fait de son succès et de la demande des externes. L'évaluation sera poursuivie.

Abstract - Background: In 2007, the department of general practice (GP) of Tours has tested a 2 weeks full time training in GP for all third year medical students of the second cycle of the medical course. The aim of this study was to examine the student's life experiences during their training. Material and methods: A qualitative study based on 4 focus groups comprising 29 students out of 104 students who did the training between July and September 2007, examined their life experiences. A complete written retranscription and an interpretative verbatim analysis were performed. Results: The training form and length were appreciated. Students discovered the specialty itself, its diversity as well as the ambulatory practice. They understood GP specificities such as: a global approach, doctor-patient relationship, ambulatory diagnostic procedure and home visit. This training was perceived useful for 
students' career orientation, allowing them to make a choice based on reality. Conclusion: This training was well experienced by students. Our study showed their lack of knowledge about GP and ambulatory practice. If human conditions and material are gathered together, this first successful experience will be repeated. Assessment will also be addressed.

\section{Introduction}

En France, dès 1997, un arrêté relatif à la deuxième partie du deuxième cycle des études médicales prescrivait en principe à chaque faculté de médecine d'organiser un stage en médecine générale ${ }^{[1]}$. Cependant, en 2006, hormis des expérimentations ponctuelles, aucun stage « grandeur nature » n'avait été mis en place dans les facultés de médecine françaises, du fait de l'absence de publication de décret d'application, de mise à disposition de ressources adéquates (financement, de moyens humains), ce qui illustrait implicitement un déficit de reconnaissance de la spécificité de la médecine générale en tant que discipline d'enseignement. La formation des étudiants à la médecine générale se résumait le plus souvent à un stage dit « de découverte» au cours du premier cycle et à quelques heures d'enseignement théorique en premier ou en deuxième cycle.

Malgré son intégration comme discipline spécifique, dotée d'un programme de formation autonome en troisième cycle et proposée en tant que telle au choix des étudiants à l'issue des épreuves classantes nationales (ECN) à partir de 2004, la médecine générale reste toujours peu attractive en France : les étudiants délaissent cette filière ou la choisissent par défaut. Plusieurs travaux, effectués dans divers pays, permettent de soutenir l'hypothèse qu'un stage en médecine générale, proposé précocement aux étudiants pour leur permettre de découvrir la spécialité, limiterait cette désaffectation ${ }^{[2-4]}$.

Dans ce contexte, les enseignants et internes en médecine générale ont fait grève en octobre 2006 pour réclamer la création d'une « filière universitaire », permettant l'identification de la discipline précocement et tout au long du cursus. Par la suite, un nouvel arrêté, promulgué le 23 novembre $2006^{[5]}$, a entériné l'organisation d'un stage en médecine générale au cours de l'externat.
En amont de cette émulation nationale, début 2006, les enseignants du département de médecine générale de la faculté de Tours ont conçu le projet d'un tel stage. La région Centre, à laquelle appartient la faculté de Tours, possède de nombreuses zones classées «médicalement déficitaires » par l'Union régionale des caisses d'assurance maladie. En contrepartie du principe de privilégier la réalisation du stage chez des médecins exerçant dans ces zones, une subvention du Fond d'action régional pour la qualité des soins de ville (FAQSV) a été obtenue. Ce financement a permis la réalisation d'un stage pour les 104 externes en troisième année du deuxième cycle des études médicales (DCEM3), de juillet à septembre 2007. Ils ont été accueillis pendant deux semaines, à temps plein, par des médecins généralistes spécifiquement formés, intervenant en tant qu'enseignants cliniciens ambulatoires (ECA). Les lieux de stage étaient répartis sur l'ensemble de la région Centre. Les externes et les ECA ont été rémunérés.

Le présent travail s'inscrit dans le cadre de l'évaluation complète de cette expérimentation qui était prévue. Plus spécifiquement, l'objectif principal de l'étude était d'explorer et de décrire l'expérience vécue par les externes au cours de leur stage en médecine générale. L'objectif secondaire était, à partir de l'exploitation de ces données, d'améliorer le stage pour les promotions suivantes.

\section{Matériel et méthodes}

Le travail rapporté est une enquête qualitative recourant à des groupes de discussion focalisée (focus groups) pour recueillir le vécu des externes. Le concept de « vécu» est compris ici dans son sens commun, tel qu'il est défini dans les dictionnaires généraux, qui désigne l'expérience réellement 
vécue, les faits, les événements de la vie. Il s'agissait notamment d'identifier ce que les externes avaient découvert, apprécié et regretté pendant leur stage.

\section{Planification du stage}

L'objectif du stage était d'exposer les étudiants à une expérience authentique de la médecine générale, dans le but qu'ils en comprennent les spécificités d'exercice.

Les ECA ont été recrutés parmi les médecins généralistes de la région Centre et spécifiquement formés à l'accueil d'externes. La plupart n'avaient jamais été maîtres de stage auparavant. La formation durait une journée. Ses objectifs étaient de : a) rappeler le cursus des études médicales et situer le niveau académique des étudiants de DCEM3 concernant leurs connaissances théoriques; b) expliciter les valeurs et spécificités de la médecine générale à illustrer à un étudiant de deuxième cycle ; c) donner quelques outils conceptuels indispensables à la transmission d'informations dans le cadre d'une démarche d'enseignement; d) expliquer les principes de la supervision pédagogique en situation d'observation directe à partir d'une mise en situation des futurs ECA (jeux de rôle); e) présenter les modalités d'évaluation des externes (cas clinique ayant trait à une situation complexe mais courante en médecine générale, exploré par quelques questions à réponse ouverte et courte, avant et après le stage). Tous les étudiants ont été convoqués pour une réunion en mai 2007, afin de recevoir des informations concernant les modalités de choix des ECA et d'évaluation de leur stage. Le stage a eu lieu de juillet à septembre 2007 ; il a consisté pour chaque étudiant à accompagner à temps plein pendant 15 jours un ECA dans ses activités professionnelles.

\section{Organisation des groupes de discussion}

Lors de la réunion d'information, le projet de cette étude a été expliqué aux étudiants. Chaque externe a été invité à s'inscrire sur une liste en vue de la participation à un groupe de discussion, dans le cadre d'une démarche volontaire, en laissant ses coordonnées. Ils ont été informés que les échanges effectués dans le cadre des groupes de discussion seraient enregistrés, l'anonymat étant respecté et la participation rémunérée à hauteur de $20 €$.

Après le stage, un courriel a été envoyé aux volontaires pour qu'ils s'inscrivent aux dates prévues pour les groupes de discussion. Initialement cinq dates avaient été proposées. Les inscriptions ont été limitées à neuf participants par séance.

Un guide d'entretien du groupe de discussion a été élaboré à partir des résultats d'une enquête quantitative préalable ${ }^{[6]}$, qui avait exploré les attentes des externes à l'égard de ce stage. Il comportait six questions ouvertes :

1. Quelle est votre opinion générale, votre ressenti global de ce stage ? En quelques mots, comment l'avez-vous vécu?

2. Que vous a-t-il apporté sur le plan personnel ?

3. Sur le plan de votre cursus, que vous a-t-il apporté par rapport aux stages hospitaliers? Et que regrettez-vous (éventuellement)?

4. Dans quelles mesures ce stage pourrait-il avoir une influence sur l'image que se font les externes de la médecine générale?

5. Dans quelles mesures pourrait-il modifier le choix professionnel futur des externes et la décision prise à l'issue des ECN?

6. Avez-vous quelque chose à ajouter ?

Les groupes de discussion ont été animés par un modérateur et un animateur. Le modérateur a pris des notes sur les aspects non verbaux des échanges et la succession des prises de parole des participants. Il a recentré la discussion si nécessaire, en reformulant les idées importantes qui émergeaient. L'animateur avait pour rôle de lancer la discussion et de distribuer la parole. Il a vérifié que tous les thèmes identifiés dans le guide d'entretien avaient bien été abordés et s'est occupé du bon fonctionnement des deux lecteurs-enregistreurs. Au début des séances, un tour de table de présentation a été effectué, au 
Tableau I. Dates, horaires et durées des quatre groupes de discussion focalisée (focus groups - FG).

\begin{tabular}{ccccc}
\hline Nom & Nombre de participants & Date & Heure & Durée \\
\hline FG1 & 9 ( 8 femmes -1 homme $)$ & $25 / 09 / 2007$ & $16 \mathrm{~h} 00$ & $120 \mathrm{~min}$ \\
\hline FG2 & 9 ( 8 femmes -1 homme $)$ & $26 / 09 / 2007$ & $16 \mathrm{~h} 00$ & $88 \mathrm{~min}$ \\
\hline FG3 & 6 (5 femmes -1 homme $)$ & $01 / 10 / 2007$ & $19 \mathrm{~h} 00$ & $86 \mathrm{~min}$ \\
\hline FG4 & 5 (3 femmes -2 hommes $)$ & $11 / 10 / 2007$ & $14 \mathrm{~h} 00$ & $80 \mathrm{~min}$ \\
\hline
\end{tabular}

cours duquel les externes ont précisé leurs projets professionnels respectifs actuels et le lieu de leur stage.

Traitement et analyse des données

Après chaque séance, une transcription tapuscrite intégrale des échanges verbaux et non verbaux a été faite. Une analyse thématique du contenu des entretiens a ensuite été réalisée en plusieurs étapes : a) une appréhension globale du contenu par plusieurs lectures attentives; b) un découpage du texte avec relevé systématique des citations et des thèmes auxquels elles se rapportent; c) un classement des thèmes; d) une interprétation dégageant les concepts abordés et les opinions exprimées. L'analyse des données a été réalisée par un chercheur dans le cadre d'un travail de thèse pour le diplôme d'état de docteur en médecine. Une validation ultérieure par un enseignant du département universitaire de médecine générale, expert en recherche qualitative, a été réalisée. En raison de contraintes liées à l'emploi du temps académique des étudiants, il n'a pas été prévu de validation de l'analyse effectuée par les étudiants ayant participé à l'étude.

\section{Résultats}

\section{Caractéristiques des groupes de discussion}

Sur les 68 externes qui s'étaient déclarés volontaires à l'issue de la réunion d'information, 32 ont répondu au courriel et se sont inscrits à l'une des dates; 29 étudiants ont finalement participé à l'étude et quatre groupes de discussion ont été organisés. Les entretiens ont été transcrits très rapidement après chaque séance, afin de ne perdre aucune donnée et leur analyse a été faite au fur et à mesure. La saturation des données a été obtenue à la quatrième séance. Le cinquième groupe de discussion prévu, ne comptant que trois inscrits, a été annulé. Les entretiens se sont déroulés à la faculté de médecine de Tours. Les caractéristiques techniques de ces séances sont résumées dans le tableau I.

Analyse et interprétation des verbatims des groupes de discussion

L'impression globale des externes a été variable et le stage qualifié de "génial », "amusant », "déprimant », "fatiguant », "pas stimulant intellectuellement »... Un étudiant a été « dégoûté » de la discipline. Mais, pour tous, ce stage se justifie pleinement au cours de leurs études et devrait être «incontournable ». Aucun ne regrettait de l'avoir fait.

Ils ont été étonnés de la "diversité de l'exercice ». Elle apparaît comme un atout de la spécialité. La patientèle est différente d'un médecin à l'autre : "On a les patients qui nous ressemblent finalement. ». Ils ont eu l'impression d'avoir bien été acceptés par les patients lors des consultations et ont essuyé peu de refus. Ils ont apprécié le mode d'annonce, que ce soit par une affiche dans la salle d'attente ou par une présentation orale.

Les étudiants ont observé, même sans l'aide pédagogique des ECA, les spécificités de l'exercice de la médecine générale, qu'ils ont toujours comparé au système hospitalier.

Ils ont compris la notion de "médecin de famille ». Le médecin est au plus près des patients, intégré dans la dynamique familiale et la vie locale. Il 
a une "place centrale» dans le système de santé : "Quelle que soit la spécialité qu'on fera, on aura affaire à des médecins généralistes! ». Les étudiants ont relevé le caractère "global» de l'approche en médecine générale, différente de la démarche hospitalière. La relation médecin-patient a été l'objet de nombreuses réactions. Ils ont aimé l'observer en pratique. Le médecin est un «confident » extrêmement proche de ses patients, les tutoyant, ce qui en a surpris certains : "Et ce qui m'a surpris un petit peu au début, c'est le tutoiement! ». Ils ont été étonnés de la «disponibilité » des ECA envers les patients. La prise en charge sociale des patients représente une part importante de l'activité : "Ce qui m'a frappé, c'est qu'il y avait pas mal de social! À l'hôpital, on en voit un petit peu mais pas trop. ». Certains ont regretté de ne pas avoir pu, en deux semaines de stage, avoir une idée plus précise du «suivi au long cours », qui caractérise selon eux la spécialité.

Les externes ont analysé les différentes étapes d'une consultation et la démarche diagnostique.

La consultation était gérée autour d'un "interrogatoire rapide » et efficace, exercice qui leur semblait «difficile». Il est suivi d'un examen clinique «ciblé », «adapté » ou «éclair ». La démarche diagnostique était «spécifique», jugée plus ardue. Elle s'inscrivait dans la durée : "C'est vraiment ce côté anti-urgence de la médecine générale! ». L' «arbre diagnostique est plus large » qu'en milieu hospitalier. La prise de décision est adaptée à la prévalence des pathologies en médecine générale. Certains étudiants ont par contre eu l'impression qu'elle était faite « au feeling ».

La coordination des soins et la gestion des examens complémentaires en milieu libéral étaient différentes de ce qu'ils connaissaient en milieu hospitalier : "On a plus les moyens à l'hôpital! (... Les examens, on les a sous la main! (...) C'est vrai que là chez le médecin généraliste, du coup, on est obligé de se limiter (...) à l'essentiel! Au strict nécessaire...».

Ils ont été "impressionnés » par les aptitudes de leurs ECA à gérer les consultations dans leur durée. Cette gestion spécifique du temps n'existe pas à l'hôpital. La consultation de renouvellement d'ordonnance a suscité beaucoup de réactions, soit d'ennui, soit d'intérêt pour l'observation de la « démarche globale de soin» et du "contact avec les patients ».

Les visites à domiciles ont été appréciées par tous les externes, sauf une. Elles étaient, pour elle, "déprimantes », en révélant la misère des patients, invisible à l'hôpital. Mais elles permettaient d' «adapter la démarche » et ont été vécues comme un moment privilégié dans la relation médecinpatient : "En visite, je trouve qu'on voit vraiment le côté humain, enfin le côté relationnel du médecin généraliste. ».

Mais les étudiants ne se sont pas limités à observer les spécificités de la discipline «médecine générale », répondant ainsi aux consignes formulées pour le stage. Leur vécu a été beaucoup plus riche. Ils ont su observer, critiquer, se projeter dans leur pratique future.

Les externes, au vu de leurs connaissances théoriques, ont parfois été très critiques sur les prescriptions (examens complémentaires, médicaments et arrêts de travail) de leurs ECA. Les patients leur paraissaient peu demandeurs d'examens. Par contre, ils réclamaient des traitements symptomatiques pour des pathologies virales bénignes. Les médecins avaient « $d u$ mal à dire non » à «ces patients qu'ils connaissent trop bien ».

Ils se sont intéressés aux activités de formation médicale continue, suivies par leurs ECA et ont discuté leur pertinence. La visite médicale des délégués pharmaceutiques a été dénigrée.

Ils ont découvert l'exercice libéral, pour la première fois au cours de leurs études. Le statut libéral constituait, pour eux, un atout de la spécialité : «C'est le point fort! », un certain « confort » de vie, qui rimait avec «liberté». Cette liberté restait relative : «On n'est pas si libre que ça! » et de nombreuses contraintes ont tout de même été relevées. Les étudiants ont observé la qualité de vie et les horaires de leurs ECA. L'emploi du temps paraissait «chargé » mais modulable et adaptable à une vie de famille : "Je pense qu'on peut, même en campagne, 
dans une zone désertée (...) organiser sa vie...». Ils ont abordé la notion de continuité des soins en observant l'organisation des systèmes de garde, la gestion des absences et la difficulté à trouver des remplaçants. L'exercice libéral, c'était aussi le paiement à l'acte. C'était quelque chose de nouveau pour eux, les mettant «assez mal à l'aise » et source de difficultés pour «réclamer». Ils pensaient y avoir été «confrontés violemment », du fait de la méconnaissance de cette relation pécuniaire à l'hôpital mais aussi en raison de l'aspect «commerce » qui s'en dégageait. Ils ont été étonnés de la confiance des patients, laissant leur médecin remplir lui-même le chèque. Ils se sont intéressés au domaine de la fiscalité et ont interrogé leurs ECA sur les charges et la comptabilité du cabinet.

Le stage a été l'occasion de découvrir le fonctionnement et la gestion d'un cabinet médical. Les étudiants préféraient les associations qui leur rappellent l'univers hospitalier et ses échanges autour des cas difficiles. Pour eux, l'association améliorait la qualité de vie des médecins généralistes. L'exercice isolé serait plus lourd. «La secrétaire, c'est indispensable ». Et les externes ont admiré leurs ECA qui arrivaient à gérer au cours de la consultation les divers appels téléphoniques. Les charges administratives étaient «chronophages » et source d'une diminution de la qualité des soins fournis. Elles devaient être déléguées. Les cabinets étaient presque tous informatisés. Les externes ont trouvé les logiciels «pratiques » d'utilisation et se les sont appropriés facilement. Ils comprenaient le frein qu'ils pouvaient être pour certains médecins, du fait « $d$ 'un temps d'adaptation » nécessaire, source de "perte de temps » au début. Un seul des quatre groupes a abordé la notion d'hygiène dans les cabinets médicaux. Pour eux, le lavage des mains n'était pas systématique mais il ne leur semblait «pas forcément justifié » et les médecins savaient «s'adapter » à chaque situation.

Les étudiants ont évoqué les conditions pratiques du stage. Ils ont apprécié la durée du stage. Deux semaines, ce n'était ni trop long, ce qui aurait été source d'ennui, ni trop court, sauf pour ceux se destinant à la médecine générale. La période à laquelle ils l'ont effectué dans leur cursus a été jugée «idéale ». Ils avaient "assez de connaissances pour suivre ce qu'il (l'ECA) faisait » et essayer «de se mettre à la place du médecin, de se dire : "Ben ça pourrait être moi! »».

Les externes ont rencontrés des difficultés de transport et de logement du fait de l'éloignement des ECA. La région étant vaste, les ECA les plus éloignés étaient à $200 \mathrm{~km}$ de la faculté.

Les étudiants se sont aussi intéressés aux conditions pédagogiques du stage. Les externes ont eu le sentiment d'avoir bien été accueillis par les ECA. Ils se sont interrogés sur le mode de recrutement des maîtres de stage et leur réelle motivation (financière, trouver un successeur, transmettre leur amour de leur métier. . .). Ils ont noté que les ECA ne connaissaient pas bien le niveau de connaissances de leur stagiaire et le sous-estimaient fréquemment. Ils regrettaient l'absence d'une liste «d'objectifs clairs». La place tenue par l'étudiant pendant la consultation a été variable. Certains sont restés assis tout au long de leur stage. Les étudiants demandaient à passer progressivement de l'observation à une participation active.

Enfin, ce stage a beaucoup apporté aux étudiants sur le plan de leur cursus universitaire et personnel. Ils ont appliqué leurs connaissances, appris à gérer un patient en ambulatoire et appréhendé la relation médecin-patient. Le stage a été vécu comme une «bouffée d'air » leur permettant de sortir du milieu hospitalier et de découvrir l'univers libéral dans lequel exercera presque la moitié d'entre eux. Il est également «utile » pour leur choix professionnel futur, confortant ou dissuadant ceux qui envisageaient de faire médecine générale et permettant aux autres d'effectuer «un choix en connaissance de cause». Ils se sont projetés dans leur pratique future et ont réfléchi à leurs conditions d'exercice.

\section{Discussion}

L'objectif principal de notre travail était d'explorer et de décrire l'expérience vécue par les externes au 
cours de leur stage en médecine générale. Pour ce faire, nous avons mis en œuvre une étude de nature qualitative. La recherche qualitative étudie les phénomènes dans leur cadre naturel. Elle s'efforce de leur donner un sens ou les interprète en faisant appel aux significations que les gens leur attribuent. Elle adopte une approche globale qui respecte la complexité des comportements humains. La technique des groupes de discussion - ou focus groups permet d'évaluer des besoins, des attentes, des satisfactions ou de mieux comprendre des opinions, des motivations ou des comportements. Elle sert aussi à tester ou faire émerger de nouvelles idées inattendues pour le chercheur ${ }^{[7]}$. Cette technique était adaptée à l'exploration du vécu de ce stage par les étudiants. Les résultats ont été riches et pour la plupart inattendus quand on les compare à la trame d'entretien. Ce travail a répondu à l'objectif fixé : décrire ce que les étudiants ont observé en pratique au cours de ce stage, ce qu'ils ont découvert, apprécié ou regretté.

Cette étude comporte cependant plusieurs limites. Ainsi, notre travail souffre d'un déficit de triangulation puisqu'un seul mode de collecte de données a été utilisé, en l'occurrence le verbatim de quatre groupes de discussion. D'autres sources d'informations, par exemple des portfolios qu'auraient pu élaborer les étudiants pendant leur stage, auraient pu être exploitées. Il est possible, par ailleurs, que les participants aux groupes de discussion (à peine un tiers des étudiants de la promotion), sélectionnés sur la base du volontariat et de la disponibilité et non pas en raison de caractéristiques ad hoc, aient eu un profil particulier. Leurs avis étaient très divergents et, dans chaque groupe de discussion, au moins un des externes n'avait pas apprécié le stage. Mais la saturation des données obtenue à la quatrième séance a montré que l'ensemble des éléments importants avaient été relevés. Nous avons cherché à limiter les conséquences du caractère peu expérimenté de l'investigateur principal par une validation ultérieure par un chercheur formé à la recherche qualitative ; en revanche, nous n'avons pas procédé à une validation par les répondants.
Une autre limite est liée au caractère purement descriptif de nos résultats. Les propos des étudiants auraient pu être interprétés dans une perspective beaucoup plus analytique. Cette limite est à notre sens acceptable compte tenu du caractère très exploratoire et préliminaire de notre travail, qui doit être rapproché du caractère en partie expérimental du stage. Ainsi, la faculté de Tours a été la première en France à proposer à une promotion entière d'externes un stage de deux semaines à plein temps en médecine générale. La bibliographie française dans ce domaine est pauvre. Une étude qualitative réalisée à la faculté de Rennes ${ }^{[8]}$ auprès de 13 externes volontaires a montré un vécu similaire au nôtre, tant sur la découverte de l'extrême diversité de la spécialité que sur les aspects relationnels et les difficultés d'exercice.

Ces deux études ont mis en évidence un point essentiel : la méconnaissance des externes français de la pratique de la médecine générale et surtout de l'univers libéral. On peut supposer que ceci résulte du déficit de formation spécifique à cet égard au cours de leur cursus antérieur et, notamment, à l'absence de stage en milieu libéral. Plusieurs études françaises non publiées concernant les opinions des étudiants ont souligné cette lacune dans la connaissance du métier de médecin généraliste et le regret de leur part de ne pas avoir été assez informés sur ce type d'exercice. En l'occurrence, le stage dont les étudiants ont fait l'expérience dans le cadre de l'étude rapportée a permis d'atteindre l'objectif fixé. Les étudiants ont compris les spécificités de la discipline et de son exercice et ce, même si, sur le plan pédagogique, les ECA n'ont pas véritablement abordé ces thématiques avec leur stagiaire. L'étude préliminaire ${ }^{[6]}$ quantitative concernant les attentes des étudiants avait montré qu'ils désiraient découvrir ces spécificités en pratique. Ils les ont toutes relevées spontanément en répondant à un questionnaire semi-ouvert. Ces notions étaient connues des étudiants de Tours en théorie et ils attendaient surtout de les observer en pratique. Les enseignements théoriques dispensés en premier et second cycle sont disparates d'une faculté à l'autre. 
Pour cette raison, les résultats de notre travail ne sont peut-être pas complètement transposables à tous les autres milieux académiques, même français. Une enquête effectuée à la faculté de médecine de Brest en 1997, ayant portée sur 89,6\% d'une promotion d'étudiants en quatrième année de médecine, avait montré que, sans avoir eu de contact formel avec ce type d'exercice au cours de leurs études, les étudiants avaient une bonne perception de ce qu'était la médecine générale et du rôle du médecin généraliste dans notre système de soins ${ }^{[9]}$. À la faculté de médecine de Rennes, le choix a été fait d'associer le stage en médecine générale à un enseignement théorique ${ }^{[8]}$ pour pallier les limites d'un compagnonnage insuffisamment explicite ${ }^{[10]}$.

Les dispositions réglementaires stipulent que le stage en médecine générale doit être organisé au cours de la deuxième partie du second cycle des études médicales, soit de la quatrième à la sixième année. Dans cette étude, les étudiants ont qualifié la fin de la cinquième année comme une période «idéale ». Comme ceux de Rennes ${ }^{[8]}$, ils pensaient que ce stage était plus profitable quand ils avaient suffisamment de recul sur les spécialités d'organe, ce qui leur a permis d'aborder plus facilement les notions de globalité et de transdisciplinarité et d'essayer de se mettre à la place de leur maître de stage.

Cette étude a aussi permis d'identifier une lacune pédagogique concernant la planification du stage, dont les objectifs n'étaient suffisamment explicites ni pour les étudiants, ni pour les ECA. Ceci explique sans doute en partie que la place accordée à l'étudiant pendant la consultation a été très variable et on peut regretter que certains aient effectué un «stage-tabouret». Il conviendra d'être attentif aux conditions de stage perçues comme défavorables, qui pourraient éventuellement desservir les finalités du stage et discréditer l'exercice de la médecine générale, comme cela a été observé pour les stages d'interne ${ }^{[11]}$.

Les étudiants ont qualifié ce stage d' « utile» pour leur choix professionnel futur, leur permettant de réaliser un « choix en connaissance de cause ». L'influence d'un stage en médecine générale à l'égard du choix d'exercice de cette discipline, que ce soit en confortant ou en remettant en cause les orientations des étudiants, a été étudiée dans le cadre de plusieurs études consacrées au recensement des principaux déterminants pour le choix de la médecine générale comme spécialité ${ }^{[2,12]}$. L'expérience d'un stage en médecine générale, avant le choix définitif de la filière, au même titre que l'existence d'un département de médecine générale au sein de la faculté ${ }^{[13]}$, semble être l'un des déterminants ${ }^{[2,3]}$ les plus importants, au regard des perceptions anticipées qu'il permet concernant la qualité de vie, la satisfaction au travail, la relation avec les patients, le suivi au long cours, la diversité de l'activité. Ce stage permet d'exposer les étudiants à un «modèle de rôle » supplémentaire, complétant ceux que jouent les chefs de service hospitaliers auprès des externes, ce qui est aussi un facteur important dans le choix ${ }^{[2]}$. Cependant, dans le cadre d'une étude impact d'un stage de quatre semaines, conduite par des enseignants britanniques ${ }^{[14]}$, un tel effet bénéfique du stage n'était que transitoire et n'était pas confirmé au moment du choix de la filière.

\section{Conclusion}

Cette étude a montré qu'au cours d'un stage de deux semaines effectué en médecine générale ambulatoire, les externes ont appréhendé les principales spécificités de l'exercice de la médecine générale et de l'univers libéral. Le stage a été bien accueilli par l'ensemble de la promotion et aucun ne regrettait de l'avoir effectué. Sur le plan de l'orientation professionnelle, le stage semble pouvoir contribuer à orienter des « choix en connaissance de cause ». Des lacunes pédagogiques concernant la planification du stage ont été mises en évidence. Elles devront être corrigées et la réflexion sur l'encadrement pédagogique doit se poursuivre. Les ECA doivent développer une compétence pédagogique appropriée au contexte de stage et, notamment, prendre conscience du fait qu'ils représentent des «modèles de rôle» accomplis sur le plan de leurs compétences cliniques. La place des étudiants et leur participation clinique 
seront définies en fonction de leur niveau de compétence. En dépit de ces limites, la moitié des externes de la promotion suivante a réclamé le renouvellement de cette expérience. Ces résultats incitent à concevoir un véritable « changement culturel dans la formation de nos étudiants ${ }^{[4]}$ pour promouvoir la spécialité. Depuis septembre 2007, un stage de six semaines est proposé au sein de notre faculté aux étudiants en quatrième année des études médicales; il est financé par des fonds débloqués par le ministère. Il n'est malheureusement accessible qu'à une très faible partie de la promotion. Des études complémentaires devront s'attacher à documenter l'impact d'un tel stage de plus longue durée.

\section{Contributions}

Cécile Renoux a participé à la conception du protocole de recherche, au recueil des données, à l'interprétation des résultats, à l'écriture du manuscrit et à la recherche bibliographique. Anne-Marie LehrDrylewicz a participé à la conception du protocole de recherche et à l'interprétation des résultats. Dominique Huas a contribué à l'écriture du manuscrit Jean-Pierre Lebeau a participé à la conception du protocole de recherche.

\section{Références}

1. Arrêté du 4 mars 1997 relatif à la deuxième partie du deuxième cycle des études médicales. NOR: MENU9700546A. Journal Officiel de la République Française, 26 mars 1997:4684-86. [On-line] Disponible sur : http: //www . legifrance.gouv. fr

2. Laurence C, Elliott T. When, what and how South Australian pre-registration junior medical officer's career choices are made. Med Educ 2007;41:467-75.

3. Mariolis A, Mihas C, Alevizos A, Gizlis V, Mariolis T, Marayiannis et al. General practice as a career choice among undergraduate medical students in Greece. BMC Med. Educ. 2007; 7:15

4. Evans J, Lambert T, Goldcrace M. GP recruitment and retention : a qualitative analysis of doctor's comments about training for and working in general practice. Occas Pap R Coll Gen Pract 2002;83:1-33.
5. Arrêté du 23 novembre 2006 pris en application de l'article 8 de l'arrêté du 4 mars 1997 modifié relatif à la deuxième partie du deuxième cycle des études médicales. NOR: SANP0624349A. Journal officiel de la République Française, 25 novembre 2006:17696. [On-line] Disponible sur : http://www. legifrance. gouv.fr

6. Renoux C, Lehr-Drylewicz AM, Robert J, Potier A, Huas D, Lebeau JP. Les attentes des externes sur le stage en médecine générale en deuxième cycle à la faculté de médecine de Tours. Exercer 2009;87:78-82.

7. Moreau A, Dedianne M-C, Letrillart L et al. Méthode de recherche : S'approprier la méthode du focus group. La Revue du Praticien Médecine Générale 2004,18:382-4.

8. Le Neel H, Honnorat C, Grosbois B, Chaperon A. Un stage en médecine générale au cours du $2^{\mathrm{e}}$ cycle : Expérimentation à la faculté de médecine de Rennes. La Revue du Praticien Médecine Générale 2007;21:407-9.

9. Pomey MP, Bail Ph, Jouquan J, Locquet C, Tison HP, Hivon $\mathrm{R}$ et al. Connaissances et croyances des étudiants en quatrième année des études de médecine à l'égard des pratiques professionnelles en médecine générale. Santé publique 1997;2:207-20.

10. Jouquan J. Pour ne pas en rester aux déclarations d'intention concernant le compagnonnage pendant l'externat. Pédagogie Médicale 2007;8:5-6.

11. Keirns C, Bosk CL. The unintended consequences of training residents in dysfunctional outpatient settings. Acad Med 2008;83:498-502.

12. Sinclair HK, Ritchie LD, Lee AJ. A future career in general practice? A longitudinal study of medical students and pre-registration house officers. Eur J Gen Pract 2006;12:120-7.

13. Kassebaum DG, Szenas PL, Schubert MK. Determinants of the generalist career intentions of 1995 graduating medical students. Acad Med 1996;71:198-209.

14. Morrison JM, Stuart Murray T. Career preferences of medical students : influence of a new four-week attachment in general practice. Br J Gen Pract 1996;46:721-5.

Correspondance et offprints : Cécile RENOUX, $31 \mathrm{~F}$ rue des 4 vents, 41350 Vineuil, France Mailto : cecilerenoux.doc@orange.fr 